\title{
Stabilized and variationally consistent nodal integration for meshfree modeling of impact problems
}

\author{
Michael Hillman · Jiun-Shyan Chen • Sheng-Wei Chi
}

Received: 15 January 2014 / Revised: 28 April 2014 / Accepted: 29 April 2014 / Published online: 23 May 2014

(C) Springer International Publishing Switzerland 2014

\begin{abstract}
Galerkin meshfree methods can suffer from instability and suboptimal convergence if the issue of quadrature is not properly addressed. The instability due to quadrature is further magnified in high strain rate events when nodal integration is used. In this paper, several stable and convergent nodal integration methods are presented and applied to transient and large deformation impact problems, and an eigenvalue analysis of the methods is also provided. Optimal convergence is attained using variationally consistent integration, and stability is achieved by employing strain smoothing and strain energy stabilization. The proposed integration methods show superior performance over standard nodal integration in the wave propagation and Taylor bar impact problems tested.
\end{abstract}

Keywords Variationally consistent integration ·

Stabilization · Integration constraint · Meshfree

\section{Introduction}

Domain integration in Galerkin meshfree methods has been a topic of interest due to the issues of stability and sub-optimal convergence which arise from the nature of the approximation functions and domain integrations employed. Meshfree

M. Hillman · J.-S. Chen $(\bowtie)$

Department of Structural Engineering, University of California, San Diego, La Jolla, CA 92093, USA

e-mail: js-chen@ucsd.edu

M. Hillman

e-mail: mhillman@eng.ucsd.edu

\section{S.-W. Chi}

Department of Civil and Materials Engineering, University of Illinois, Chicago, IL 60607, USA

e-mail: swchi@uic.edu approximation functions are in general rational, often with complicated overlapping support structures, and both characteristics contribute to quadrature inaccuracy. In the former case, quadrature schemes such as Gauss cannot provide exact integration of these functions. In the latter case, misalignment of integration cells with supports can cause a great deal of quadrature inaccuracy [12]. These problems are more severe in nodal integration methods, which present an even greater challenge for meshfree methods since they are often employed so that the character of the method is preserved.

Nodal integration methods such as direct nodal integration (DNI) can suffer from stability issues [2,9] as well as sub-optimal convergence [4,9] and often require special techniques to alleviate the problems. Beissel and Belytschko [2] showed direct nodal integration for the moving least squares approximation can result in instability due to the fact that the Galerkin equation with nodal integration gives very low energy for saw-tooth modes, resulting from zero or nearly zero derivatives at nodal points. They proposed least-squares stabilization which alleviates the problem, although the technique requires second order derivatives in the Galerkin equation as well as second order consistency of the shape functions. Liu et al. [15] introduced a Taylor series expansion approach to alleviate the instability, but requires third order derivatives. The strain smoothing stabilized conforming nodal integration (SCNI) has been proposed $[9,10]$ to ease the situation, where derivatives are not directly evaluated at nodes which avoids the instability, but still requires attention because of additional unstable modes which may become excited in certain situations [5,19].

The sub-optimal convergence of meshfree methods with improper quadrature can be attributed to Strang's first Lemma [20]. The use of quadrature in the Galerkin equation results in loss of Galerkin orthogonality and subsequently the best approximation property of the solution, and can result in con- 
vergence rates much lower than predicted by exact integration, or even solutions which diverge with refinement [4]. Typically background cell integration without higher order quadrature does not provide sufficient accuracy due to the complexity of meshfree shape functions. The approximation functions are often rational with overlapping supports, and it is difficult to provide accurate integration. In early constructions where background grids were adopted for domain integration $[3,18]$, no particular approach was taken to alleviate quadrature inaccuracy. However in [12] it was recognized that misalignment of integration cells and shape functions supports is a major source of quadrature error, and a scheme was proposed where they align allowing for restoration of convergence rates. Several methods similar in spirit have since been proposed $[1,11,17]$, which can also preserve the meshfree character of the Galerkin method but can carry a computational burden.

As an alternative approach, the SCNI method introduced in [9] uses strain smoothing for first order Galerkin exactness and recovers quadratic convergence in the $L^{2}$ norm for linear basis. SCNI has been applied to other meshfree methods [25] and has also been extended to plates and shells $[7,22,23]$. This technique was later generalized to higher order strain smoothing in [13] giving cubic rates of convergence in the $L^{2}$ norm. In the recent work in [4], the condition for arbitrary high order Galerkin exactness was derived under the general framework of variational consistency, and several variationally consistent integration (VCI) methods were proposed. Here it was shown that when variational consistency is satisfied, optimal convergence can be attained with far fewer quadrature points than would otherwise be required.

In this work, the variationally consistent integration proposed in [4] is applied to elastodynamics and geometric and material non-linear problems. The VCI methods show more favorable phase and amplitude in transient problems as well as superior performance for large deformation problems compared to their variationally inconsistent counterparts. Stabilization techniques are also employed based on the works in $[5,19]$, and an eigenvalue analysis of the combined methods is provided.

The outline of the paper is as follows. Section 2 gives a basic overview of domain integration for Galerkin meshfree methods, and demonstrates how variationally inconsistent integration methods can exhibit sub-optimal convergence and how nodal integration can lead to instability. In Sect. 3, several variationally consistent integration methods are introduced along with enhanced stabilization for nodal integration. In Sect. 4, the stabilized VCI methods are applied to several problems demonstrating improved performance over standard methods in the dynamic and large deformation setting. Concluding remarks are then given in Sect. 5.

\section{Background}

In this section we review the issues associated with domain integration in Galerkin meshfree methods. We consider the RK approximation to illustrate the characteristics of the approximations used in meshfree methods. Here it is shown how several integration methods exhibit sub-optimal convergence and instability under certain discretizations.

\subsection{Reproducing kernel (RK) approximation}

The RK approximation $u^{h}(\mathbf{x})$ of a function $u(\mathbf{x})$ is constructed as:

$u^{h}(\mathbf{x})=\sum_{I=1}^{N P} \Psi_{I}(\mathbf{x}) u_{I}$

where $\Psi_{I}(\mathbf{x})$ is the approximation function with compact support which possesses the following reproducing conditions of degree $n$ :

$$
\sum_{I=1}^{N P} \Psi_{I}(\mathbf{x}) \mathbf{x}_{I}^{\alpha}=\mathbf{x}^{\alpha}, \quad|\alpha| \leq n
$$

In the above, the multi-index notation has been introduced. Here $\alpha \equiv\left(\alpha_{1}, \alpha_{2}, \ldots, \alpha_{d}\right)$, with the length of $\alpha$ defined as $|\alpha|=\sum_{i=1}^{d} \alpha_{i}, \mathbf{x}^{\alpha} \equiv x_{1}^{\alpha_{1}} \cdot x_{2}^{\alpha_{2}} \cdot \ldots \cdot x_{d}^{\alpha_{d}}$, and $\mathbf{x}_{I}^{\alpha} \equiv x_{I 1}^{\alpha_{1}} \cdot x_{I 2}^{\alpha_{2}}$. $\ldots \cdot x_{I d}^{\alpha_{d}}$. The above function $\Psi_{I}(\mathbf{x})$ is constructed by selecting a kernel function $\Phi_{a}\left(\mathbf{x}-\mathbf{x}_{I}\right)$ with compact support with measure " $a$ " and a correction function composed of a linear combination of basis functions in the following form [16]:

$\Psi_{I}(\mathbf{x})=\left\{\sum_{|\alpha| \leq n}\left(\mathbf{x}-\mathbf{x}_{I}\right)^{\alpha} b_{\alpha}(\mathbf{x})\right\} \Phi_{a}\left(\mathbf{x}-\mathbf{x}_{I}\right)$

The terms $\left\{\left(\mathbf{x}-\mathbf{x}_{I}\right)^{\alpha}\right\}_{|\alpha| \leq n}$ are the set of basis functions and $\left\{b_{\alpha}(\mathbf{x})\right\}_{|\alpha| \leq n}$ are coefficients which are obtained by meeting (2.2). The kernel function $\Phi_{a}\left(\mathbf{x}-\mathbf{x}_{I}\right)$ determines the locality and smoothness of the approximation. For example, a cubic spline kernel function yields the approximation function in (2.3) $C_{2}$ continuous. The approximation function in (2.3) thus gives the reproduction of monomials of arbitrary degree $n$, and arbitrary smoothness of the approximation by the appropriate selection of kernel function $\Phi_{a}\left(\mathbf{x}-\mathbf{x}_{I}\right)$. These properties can be obtained without a structured mesh as described below.

By introducing (2.3) into (2.2), the coefficients $\left\{b_{\alpha}(\mathbf{x})\right\}_{|\alpha| \leq n}$ are obtained, and the RK approximation functions are defined as

$$
\Psi_{I}(\mathbf{x})=\mathbf{H}(\mathbf{0})^{\mathrm{T}} \mathbf{M}^{-1}(\mathbf{x}) \mathbf{H}\left(\mathbf{x}-\mathbf{x}_{I}\right) \Phi_{a}\left(\mathbf{x}-\mathbf{x}_{I}\right)
$$


Fig. 1 Integration schemes used for the linear patch test: DNI, $1 \times 1$ GI, SCNI, and SNNI

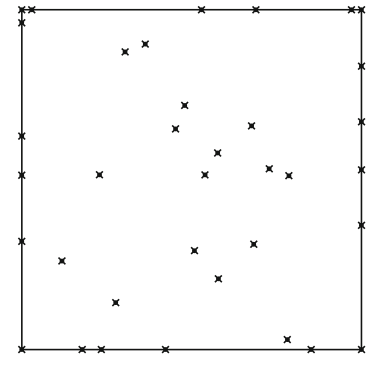

DNI

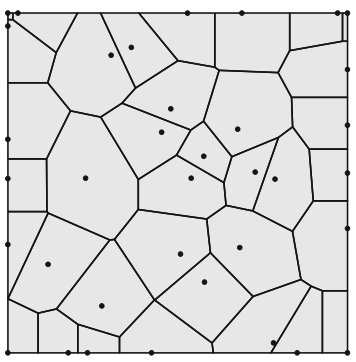

SCNI

\begin{tabular}{|c|c|c|c|c|}
\hline$x$ & $x \cdot$ & $x$ & $x$ & $x$ \\
\hline$x$ & $x$ & $\cdot^{\times}$ & $\cdot^{x}$ & $x$ \\
\hline$x$ & $\cdot x$ & $\times \cdot$ & $\times$ & $x$ \\
\hline$x$ & $x$ & $x$ & $\times$ & $x$ \\
\hline$x$ & $\times$ & $x$ & $x$ & $x$ \\
\hline
\end{tabular}

1x1 GI

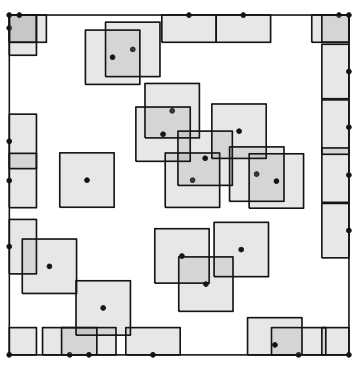

SNNI
- RKPM node

$\times$ Integration point

Gauss cell

Smoothing zone
Table $1 L^{2}$ Errors in linear patch test

\begin{tabular}{lllllllll}
\hline Method & DNI & $1 \times 1$ GI & $2 \times 2$ GI & $3 \times 3$ GI & $4 \times 4$ GI & $5 \times 5$ GI & SCNI & SNNI \\
\hline$L^{2}$ norm & 0.67501 & 1.6402 & 0.0782 & 0.02418 & 0.00668 & 0.00194 & $1.06 \mathrm{E}-14$ & 0.94592 \\
\hline
\end{tabular}

$\nabla^{2} u=0 \quad$ in $\Omega$

$\nabla u \cdot \mathbf{n}=n_{1}+2 n_{2} \quad$ on $\partial \Omega_{h}$

$u=x+2 y \quad$ on $\partial \Omega_{g}$

$\mathbf{M}(\mathbf{x})=\sum_{I=1}^{N P} \mathbf{H}\left(\mathbf{x}-\mathbf{x}_{I}\right) \mathbf{H}^{\mathrm{T}}\left(\mathbf{x}-\mathbf{x}_{I}\right) \Phi_{a}\left(\mathbf{x}-\mathbf{x}_{I}\right)$

Here the vector $\mathbf{H}^{\mathrm{T}}\left(\mathbf{x}-\mathbf{x}_{I}\right)$ is the row vector of $\left\{\left(\mathbf{x}-\mathbf{x}_{I}\right)^{\alpha}\right\}_{|\alpha| \leq n}$ and $\mathbf{M}(\mathbf{x})$ is the moment matrix. The reproducing conditions (2.2) are met provided there are sufficient points under the cover of $\Phi_{a}\left(\mathbf{x}-\mathbf{x}_{I}\right)$ so that the equations resulting from (2.2) and (2.3) are linearly independent and the moment matrix $\mathbf{M}(\mathbf{x})$ is nonsingular [6].

\subsection{Loss of best approximation property and sub-optimal convergence due to inaccurate quadrature rules}

The use of inaccurate quadrature in the Galerkin equation results in the loss of the Galerkin orthogonality and could lead to sub-optimal convergence in the Galerkin solution according to Strang's first Lemma [20]. The concept of variational consistency has been proposed as a means to correct inaccurate quadrature rules to recover Galerkin orthogonality, and consequently achieve optimal convergence in the Galerkin solution [4].

To illustrate this, consider the Poisson problem with linear solution $u=x+2 y$ : where $\Omega:(-1,1) \times(-1,1), \partial \Omega_{h}:-1 \leq x \leq 1, y=$ $1, \partial \Omega_{g}=\partial \Omega \backslash \partial \Omega_{h}$. The RK approximation with linear basis is introduced with several integration methods considered: DNI, Gauss integration (GI) with increasing order $m$ (denoted herein as $m \times m G I$ ), the first order variationally consistent method SCNI [9], and stabilized non-conforming nodal integration (SNNI) [8,14], shown in Fig. 1. The exact linear solution in the above problem is not obtained for GI, DNI, and SNNI which are variationally inconsistent, as seen in Table 1. The results show that even though the basis functions possess sufficient completeness to represent the exact linear solution, it is not obtained when inappropriate quadrature is used.

The solution error due to variational inconsistency manifests as deteriorated convergence rates. Consider again the Poisson problem, now with a higher order solution $u=$ $\sin (\pi x) \sin (\pi y) / 2 \pi^{2}$ :

$$
\begin{array}{ll}
\nabla^{2} u+\sin (\pi x) \sin (\pi y)=0 & \text { in } \Omega \\
u=0 & \text { on } \partial \Omega
\end{array}
$$

where $\Omega:(-1,1) \times(-1,1)$. Linear basis are introduced with the previous integration schemes, with the discretizations 

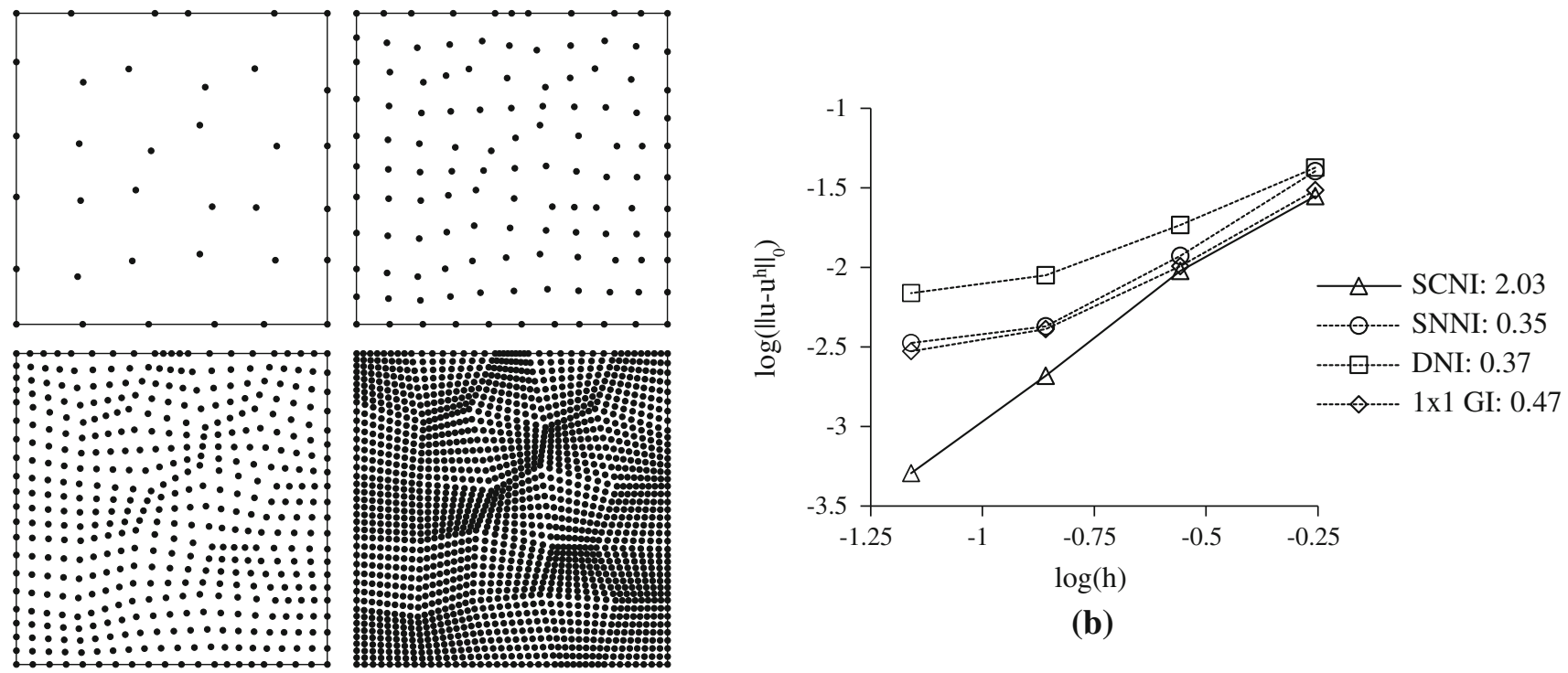

(b)

(a)

Fig. 2 a Uniform refinement of irregular node distribution, b convergence with various domain integrations, rates indicated in legend

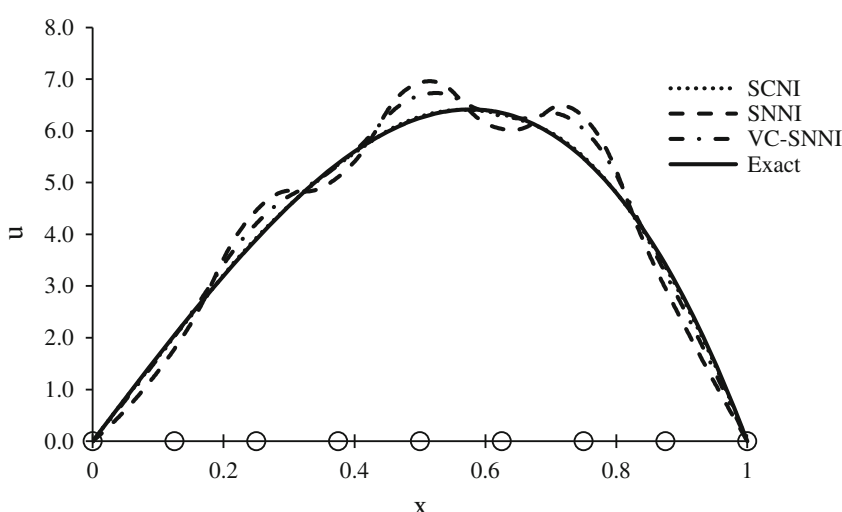

(a)

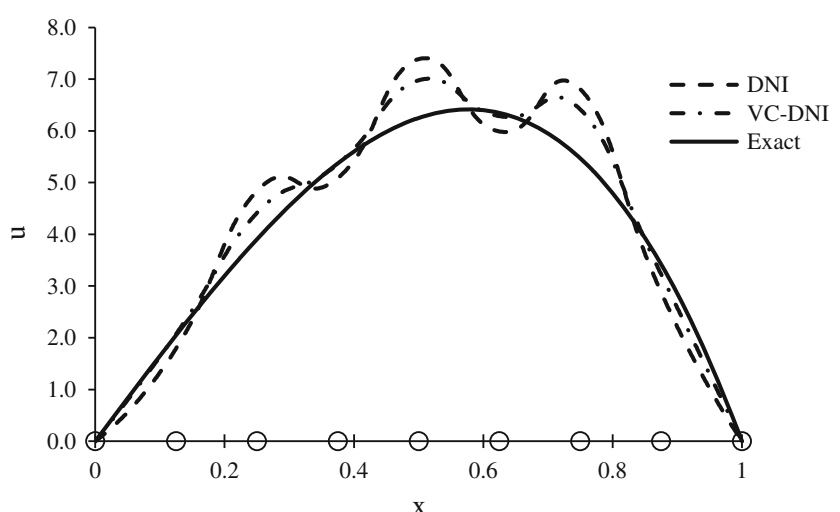

(b)

Fig. 3 Solution by a SCNI, SNNI, and VC-SNNI, and b DNI and VC-DNI. Nodal locations indicated by circles

shown in Fig. 2a. Here refinement of a non-uniform node distribution is employed, as they are particularly problematic for quadrature in meshfree methods. It is seen in Fig. $2 b$ that convergence is severely deteriorated for methods which are not first order variationally consistent. On the other hand, the first order variationally consistent SCNI method shows optimal convergence (rate of 2). In the next section, several ways to construct variationally consistent methods are reviewed.

\subsection{Nodal integration leading to instability}

When nodal integration is employed for meshfree methods, instability can arise due to the underestimation of energy associated with small wavelength modes. This is due to the fact that first order derivatives (which appear in the weak form) of the modes are zero or nearly zero at nodal locations. The SCNI method $[9,10]$ has been introduced which avoids evaluating derivatives at nodal locations, thus circumventing the issue of zero energy modes.

To illustrate, consider a one-dimensional bar with a linear body force:

$u_{, x x}+100 x=0$

$u(0)=\mathrm{u}(1)=0$

Quadratic RK basis is employed with a uniform distribution of nine nodes, and the nodal integration methods in the previous example are considered along with variational consistency (VC) corrected SNNI (VC-SNNI) and DNI (VC-DNI) [4]. All of the solutions shown in Fig. 3 exhibit the oscillatory modes associated with the instability except for SCNI. Although greater stability is provided by SNNI over DNI, the oscillatory pattern is still apparent in the solution. The results for VC-DNI and VC-SNNI show that although VCI enhances accuracy, it provides only slightly better stability, 
and additional stabilization of these methods is needed and will be introduced in the next section.

\section{Variational consistency and stabilization for nodal integration}

In this section the concept of variational consistency is reviewed, along with stabilization for nodal integration. It is shown how VCI can restore Galerkin exactness up to the order of completeness in the approximation and provide optimal convergence. Stabilization for nodal integration is introduced, and an eigenvalue analysis for nodal VCI methods with stabilization is also given.

\subsection{Variationally consistent integration}

The concept of variational consistency introduced in [4] can be used as a guideline to construct quadrature schemes and test functions consistent with each other. The variational consistency condition is a generalization of the integration constraint for linear exactness given in [9], where it has been extended to arbitrary high order solutions.

For first order variational consistency, it was first shown in [9] that in addition to having first order completeness in the approximation, the following integration constraint in the form of a divergence condition must be satisfied for the quadrature rule employed:

$$
\int_{\Omega}^{\wedge} \nabla \tilde{\Psi}_{I}(\mathbf{x}) \mathrm{d} \Omega=\int_{\partial \Omega}^{\wedge} \tilde{\Psi}_{I}(\mathbf{x}) \mathbf{n} \mathrm{d} \Gamma \quad \forall I
$$

Here, the superposed " $\wedge$ " denotes the numerical integration, $\tilde{\Psi}_{I}(\mathbf{x})$ is the test function, and $\mathbf{n}$ is the unit outward surface normal. The above equation states that the quadrature rule used in the Galerkin equation should be consistent with the test function to achieve Galerkin exactness. If nodal integration is employed, strain smoothing proposed in $[9,10]$ can be adopted to meet the first order integration constraint:

$$
\begin{aligned}
\widetilde{\nabla} u^{h}\left(\mathbf{x}_{\mathbf{L}}\right) & =\frac{1}{A_{L}} \int_{\Omega_{L}} \nabla u^{h}(\mathbf{x}) \mathrm{d} \Omega \\
& =\frac{1}{A_{L}} \int_{\partial \Omega_{L}} u^{h}(\mathbf{x}) \mathbf{n}(\mathbf{x}) \mathrm{d} \Gamma
\end{aligned}
$$

where $A_{L}=\int_{\Omega_{L}} d \Omega$ and $\Omega_{L}$ is the conforming representative domain of point $L$ as shown in Fig. 4a. Conforming cells can be generated using Voronoi diagrams or Delaunay triangulation. The strain smoothing in (3.2) is the basis of SCNI $[9,10]$, which results in linear exactness and quadratic convergence in the $L^{2}$ norm. More recently, similar assumed strain constructions have been proposed for second order variationally consistency in [13].

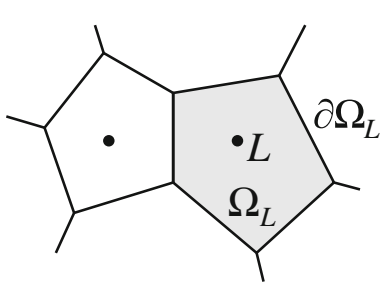

(a)

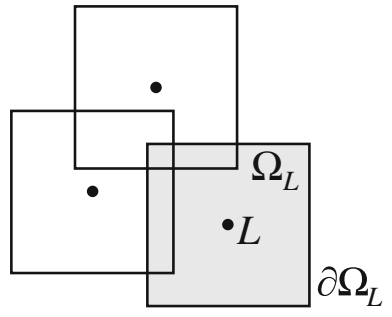

(b)
Fig. 4 Nodal representative domain for a SCNI and $\mathbf{b}$ SNNI

Simplifications of SCNI for extremely large deformation problems have been proposed such as stabilized nonconforming nodal integration (SNNI) $[8,14]$. Here the smoothing zones are simply cells constructed around the nodes with the conforming condition relaxed, as shown in Fig. 4b. As a consequence, the integration constraint is no longer satisfied and sub-optimal convergence is encountered as shown in Fig. 2.

A general framework for achieving $n$th order Galerkin exactness is the work in [4], which generalizes the condition in (3.1) to $n$th order integration constraints obtained by introducing $n$th order variational consistency and completeness of the trial functions:

$$
\begin{aligned}
a\left\langle\tilde{\Psi}_{I}, \mathbf{x}^{\alpha}\right\rangle_{\Omega}= & -\left\langle\tilde{\Psi}_{I}, L \mathbf{x}^{\alpha}\right\rangle_{\Omega} \\
& +\left\langle\tilde{\Psi}_{I}, B \mathbf{x}^{\alpha}\right\rangle_{\partial \Omega} \forall I, \quad|\alpha|=0,1, \cdots, n
\end{aligned}
$$

where $a\langle\cdot, \cdot\rangle_{\Omega}$ is the quadrature version of the bilinear form, $\langle\cdot, \cdot\rangle_{\Omega}$ and $\langle\cdot, \cdot\rangle_{\partial \Omega}$ are the quadrature versions of inner products of two functions in the domain and on the boundary, respectively, and $L$ and $B$ are the differential operator and the Neumann boundary operator of the boundary value problem, respectively. The condition in (3.3) states that in order to achieve $n$th order Galerkin exactness, the test functions must be consistent with the numerical integration following (3.3). These requirements are in the form of an integration-by-parts type formula which depend on the boundary value problem at hand. If exact integration is employed one can observe that (3.3) is automatically satisfied and Galerkin exactness can be obtained provided the approximation space possesses $n$th order completeness. In general, most integration schemes for meshfree methods (e.g. DNI, GI, or SNNI) do not satisfy (3.3) with the exception of a few, including SCNI for $n=1$.

As an alternative to strain smoothing, modified test functions can be introduced into the Galerkin equation to satisfy the integration constraint. The procedure proposed in [4] is to construct test functions which are variationally consistent with a given integration method. A correction to the test function is introduced in the following manner: 
$\tilde{\Psi}_{I}(\mathbf{x})=\Psi_{I}(\mathbf{x})+\sum_{|\beta| \leq n} \xi_{\beta I} \tilde{\Psi}_{I}^{\beta}(\mathbf{x})$

Here it is required that $\left\{\Psi_{I}, \tilde{\Psi}_{I}^{\beta}\right\}_{|\beta|=1}^{n}$ are linearly independent. Inserting the above test functions into the integration constraint (3.3) yields

$$
\sum_{|\beta|=1}^{n} A_{\alpha \beta I} \xi_{\beta I}=r_{\alpha I} \quad \forall I, \quad|\alpha|=0,1, \cdots, n
$$

where $A_{\alpha \beta I}$ and $r_{\alpha I}$ are the components of the resulting linear system matrix and residual vector, respectively. In the above equation, the right hand side represents the violation of integration constraints and the coefficients solved from (3.5) correct the violation. Here it can be seen that the correction is driven directly by the residual, and for variationally consistent methods no correction is needed, due to zero residual. The resulting method is arbitrary order variationally consistent, and only requires the solution to relatively small linear systems.

The system in (3.5) can also be decoupled by considering the gradient approximations:

$\nabla u^{h}(\mathbf{x})=\sum_{I=1}^{N P} \nabla \Psi_{I}(\mathbf{x}) u_{I}$,

$\tilde{\nabla} v^{h}(\mathbf{x})=\sum_{I=1}^{N P}\left(\nabla \Psi_{I}(\mathbf{x})+\sum_{|\beta| \leq n} \xi_{\beta I} \nabla \tilde{\Psi}_{I}^{\beta}(\mathbf{x})\right) v_{I}$

As an example, consider the corrected gradient for the linear integration constraint for the 2-dimensional Poisson and elasticity equations:

$$
\begin{aligned}
& \tilde{\nabla} v^{h}(\mathbf{x})=\sum_{I=1}^{N P}\left(\nabla \Psi_{I}(\mathbf{x})+R_{I}(\mathbf{x})\left\{\begin{array}{l}
\xi_{1 I} \\
\xi_{2 I}
\end{array}\right\}\right) v_{I}, \\
& R_{I}(\mathbf{x})=\left\{\begin{array}{l}
1 \text { if } \mathbf{x} \in \operatorname{supp}\left(\Psi_{I}(\mathbf{x})\right) \\
0 \text { if } \mathbf{x} \notin \operatorname{supp}\left(\Psi_{I}(\mathbf{x})\right)
\end{array}\right.
\end{aligned}
$$

With the above construction, first order Galerkin exactness is restored, and optimal convergence can be achieved. Consider the integration methods described in Sect. 2.2 with the problem in (2.7) and discretizations in Fig. 2a. RK approximations with linear basis are introduced with the correction in (3.7) employed for variationally inconsistent methods (with the resulting GI method denoted as $1 \times 1 \mathrm{VC}-\mathrm{GI}$ ). It can be seen in Fig. 5 that the convergence is superior for VCI methods over their standard counterparts, and optimal in most cases. Arbitrarily high order corrections can be obtained in a similar manner, for elaborations, see [4].

\subsection{Additional stabilization for nodal integration}

Non-zero energy oscillatory modes exist in SCNI when the surface area to volume ratio is small. In the same situation,

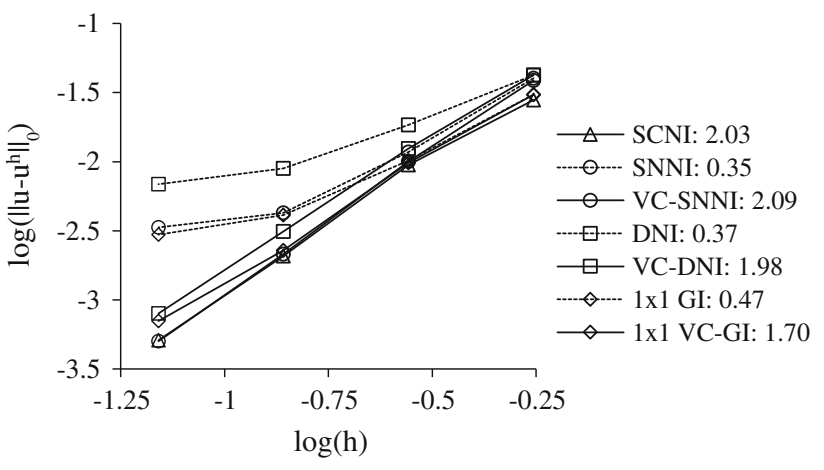

Fig. 5 Convergence of integration methods with and without variational consistency

similar modes also exist in SNNI. Short-wavelength modes associated with only a small amount of energy initiated from the boundary may become excited. When the discretization is fine, or when the volume is comparatively larger than the surface area, these modes remain relatively unchecked [19].

Consider the discretization shown in Fig. 6a with SCNI and SNNI employed for numerical integration. Eigenvalue analysis of the 2-D elastic stiffness matrix reveals that the lowest non-zero energy modes are oscillatory, as seen in Fig. 6 b.

The stabilization of these modes can be accomplished by an additional bilinear term which enhances coercivity. Strain averaging is employed using a subdivision of the smoothing cells, where additional stabilization points are evaluated. The form is based on maintaining satisfaction of patch test for SCNI, and uses the strain averaging as a limiter for unstable modes [19]:

$$
\begin{aligned}
& a\left(\mathbf{v}^{h}, \mathbf{u}^{h}\right)=\sum_{L=1}^{N P} \tilde{\varepsilon}_{L}\left(\mathbf{v}^{h}\right): \mathbf{C}: \tilde{\varepsilon}_{L}\left(u^{h}\right) A_{L} \\
& \quad+\sum_{L=1}^{N P} \sum_{K=1}^{N S} c\left[\left(\tilde{\varepsilon}_{L}\left(v^{h}\right)-\varepsilon_{K}\left(v^{h}\right)\right): \mathbf{C}:\left(\tilde{\varepsilon}_{L}\left(u^{h}\right)-\varepsilon_{K}\left(u^{h}\right)\right) A_{K}\right]
\end{aligned}
$$

where $N S$ is the number of stabilization points, $\tilde{\varepsilon}_{L}$ is the smoothed strain at node $L, \varepsilon_{K}$ is the strain at stabilization point $K, \mathbf{C}$ is the matrix of material constants, $c$ is a stabilization parameter ranging from zero to unity, and $A_{K}$ is the cell area associated with point $K$. Note that for SNNI, the weights for stabilization points are taken as $A_{L} / N S$. The distribution of points $K$ in relation to node $L$ for SCNI and SNNI is depicted in Fig. 7. In (3.8), the second term is the contribution of the stabilization; in explicit dynamics it leads to an additional internal force term.

An eigenvalue analysis is performed on the stiffness matrices associated with the discretization in Fig. 6a with the additional stabilization (3.8) with $c=0.1$, for modified SCNI (MSCNI) and modified SNNI (MSNNI). The 
Fig. 6 a Discretization, b low energy modes and eigenvalues of SCNI and SNNI (two modes)
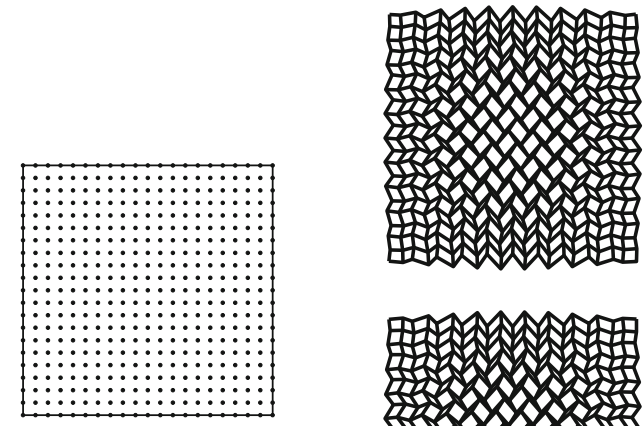

(a)

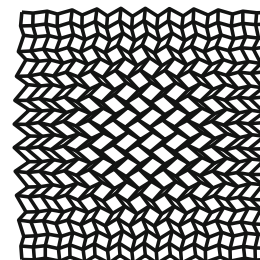

SNNI:0.743
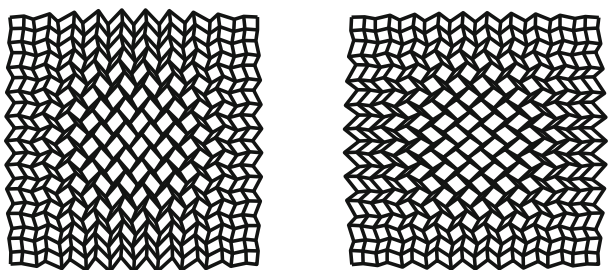

SCNI:0.739
Fig. 7 Stabilization schemes for a SCNI and $\mathbf{b}$ SNNI

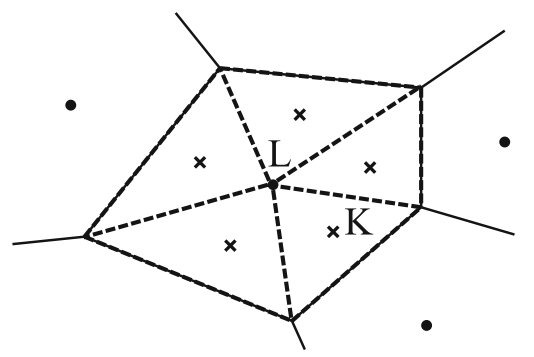

(a)

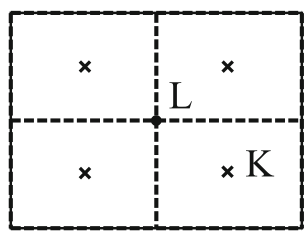

(b)

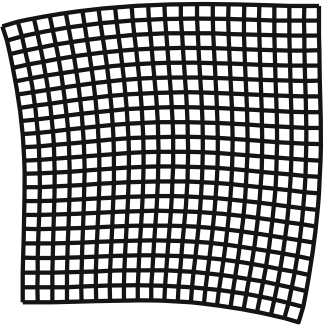

MSCNI:1.263

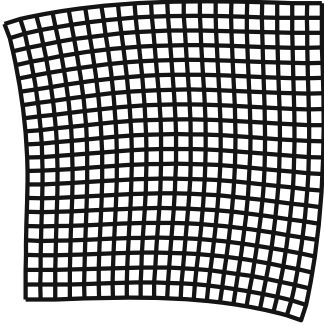

MSNNI:1.278

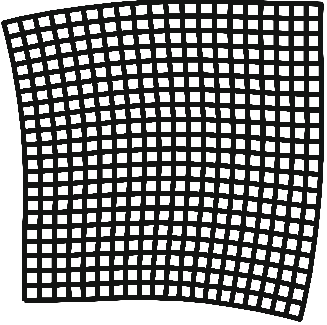

FEM: 1.300
- RK node

$\times$ stabilization point

$\square$ smoothing zone

L-I stabilization cell

Fig. 8 Lowest energy modes eigenvalues of MSCNI, MSNNI, and fully integrated FEM

lower energy modes are now stable modes of deformation, and the lowest eigenvalue and eigenmode match well with fully integrated linear FEM elements, as seen in Fig. 8. Here it is noted, while not shown in Fig. 8, that VC-SNNI gives similar results to SNNI, and VC-MSNNI yields similar results to MSNNI indicating that the correction by variational consistency is marginal in uniform discretization.

When the non-uniform discretization shown in Fig. 9 is analyzed, it is shown that the VC correction provides stabilization. This can be seen in the significant improvement of the lowest eigenvalue and eigenmode in VC-SNNI over SNNI. It is also seen that in this case SCNI does not exhibit instability due to the irregular spacing of nodes, and the sta- bilization gives only slightly better eigenvalues. For SNNI, some oscillations are observed, while MSNNI provides more stability. VC-MSNNI gives much better values than its variationally inconsistent counterpart, again indicating that VCI provides additional stability.

\section{Numerical examples}

In this section numerical examples are provided showing superior performance of VCI and VCI with additional stabilization. Linear static and dynamic problems are solved, as well as a non-linear large deformation problem. The nomenclature adopted for the numerical methods tested are listed in Table 2. 


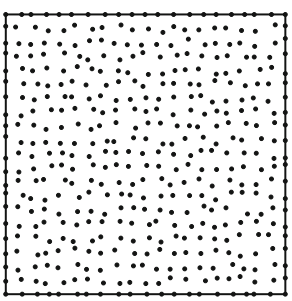

Discretization

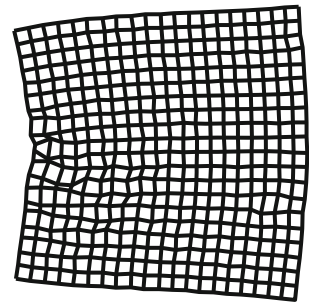

SNNI: 0.747

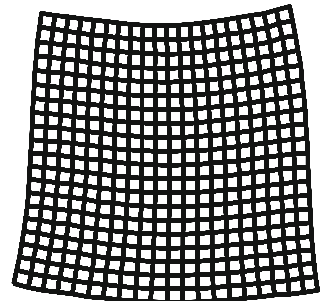

FEM: 1.296

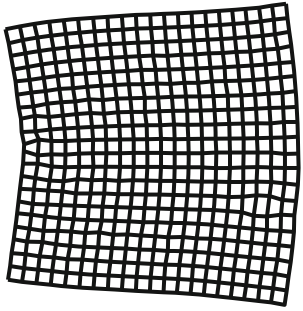

MSNNI: 0.963

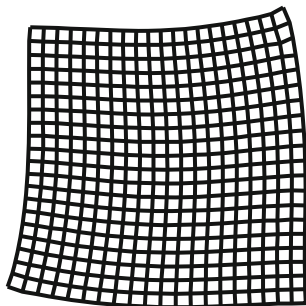

SCNI: 1.246

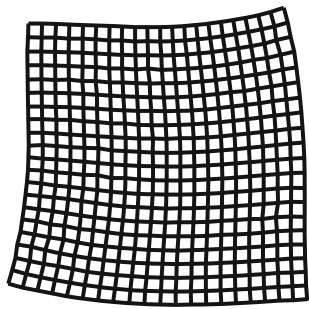

VC-SNNI: 1.201

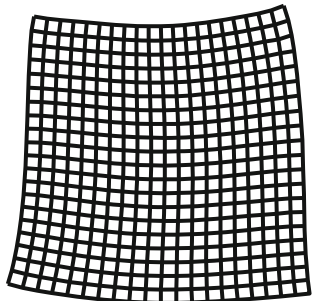

MSCNI:1.256

Fig. 9 Comparison of lowest energy modes and eigenvalues for stabilization of SCNI, stabilization and correction of SNNI, and fully integrated linear FEM

Table 2 Nomenclature for domain integration

\begin{tabular}{llll}
\hline Standard & Stabilized & VC corrected & $\begin{array}{l}\text { Stabilized and } \\
\text { VC corrected }\end{array}$ \\
\hline SCNI & MSCNI & - & - \\
SNNI & MSNNI & VC-SNNI & VC-MSNNI \\
DNI & - & VC-DNI & - \\
\hline
\end{tabular}

\subsection{Tube problem}

Consider the infinitely long tube system shown in Fig. 10 with material properties Young's modulus $E=3.0 \times 10^{7}$ and Poisson's ratio $v=0.3$. The tube has the dimensions outer radius $R=1.0$ and thickness $T=0.5$, and is subject to an internal pressure $p=1.0 \times 10^{7}$. The integration methods SCNI, SNNI, and VC-SNNI are applied to the discretizations shown in Fig. 11a with linear basis and cubic B-spline kernel introduced for the RK approximation. It can be seen that SCNI and VC-SNNI methods converge optimally, while SNNI nearly stops converging with refinement as seen in Fig. 11b.

\subsection{Wave propagation in an elastic bar}

Consider an elastic bar with $x \in[0.0,20.0]$ constrained at $x=0.0$ subjected to an initial velocity of $v_{0}=1.0$. Material parameters for the bar are $E=1.0 \times 10^{2}, v=0.3$, and density $\rho=1.0$. The RK approximation with linear basis and cubic B-spline kernel function is introduced with DNI, VC-DNI, $1 \times 1$ GI, $1 \times 1$ VC-GI, SCNI, SNNI and

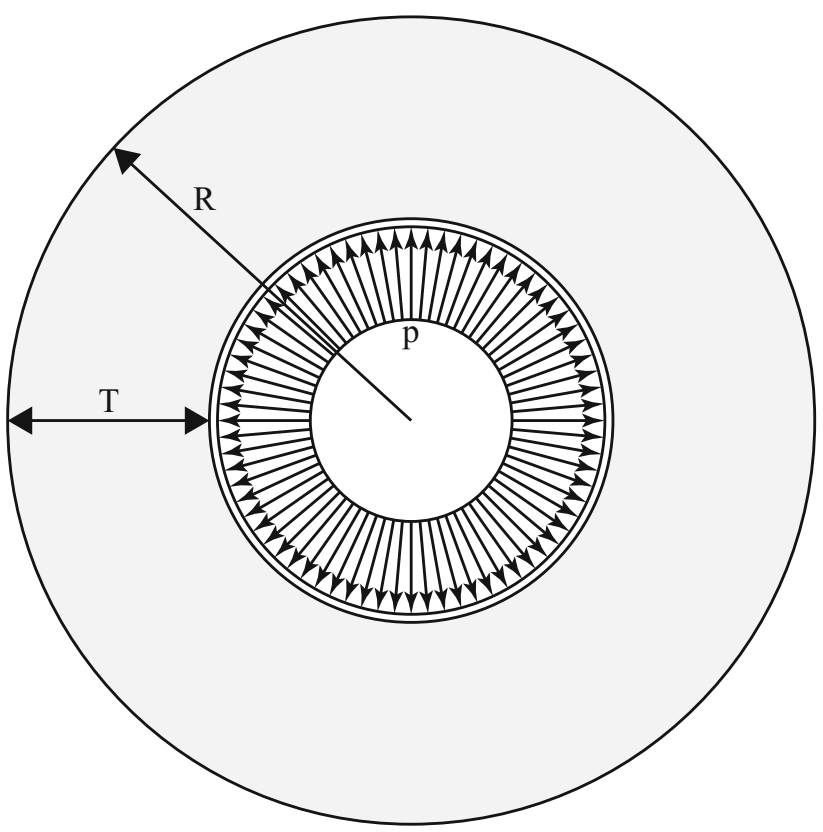

Fig. 10 Linear elastic tube system

VC-SNNI employed for domain integration, and the nonuniform node distribution shown in Fig. 12 is considered. Due to the non-uniform discretization employed, additional stabilization is not considered as motivated by the results in Sect. 3.2. Here, central difference time integration with lumped mass is employed. Methods which are variationally inconsistent exhibit phase as well as amplitude errors in the solution, while the VCI methods show superior performance 

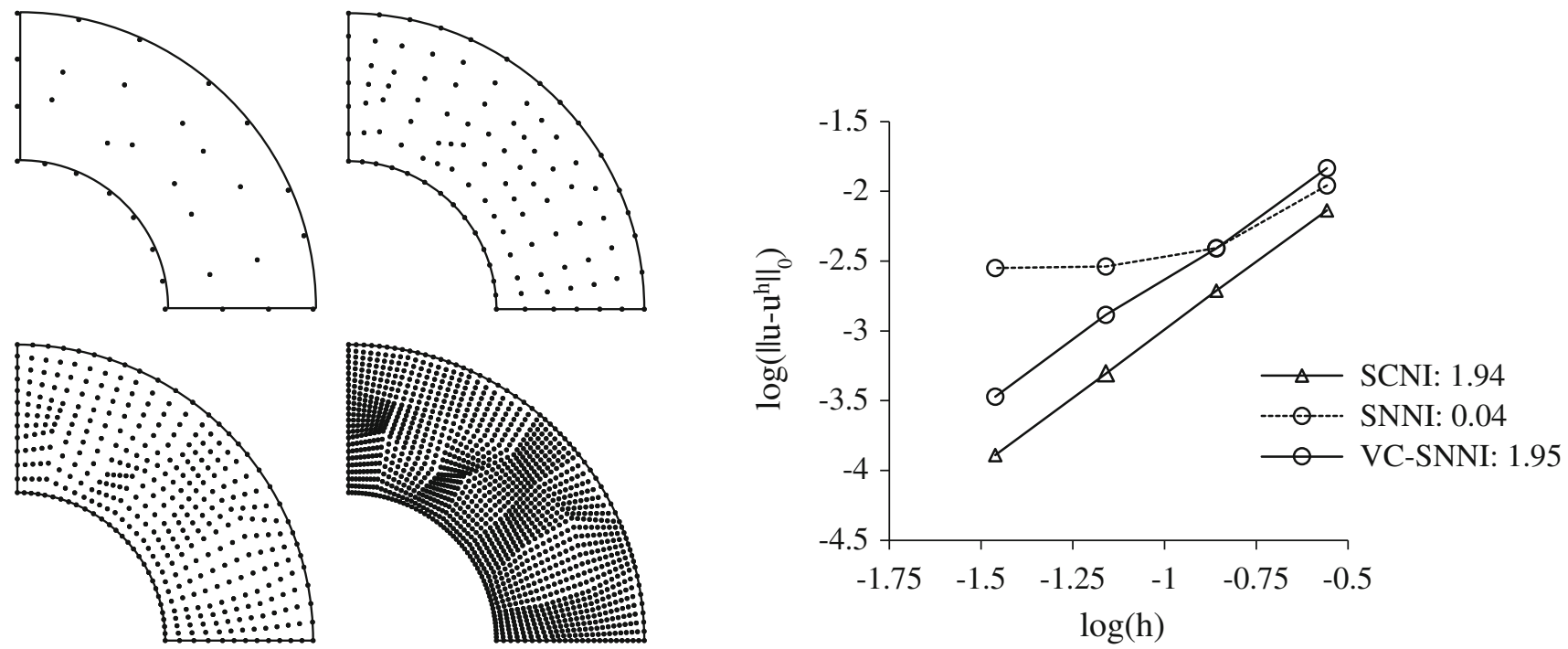

(a)

(b)

Fig. 11 a Tube discretizations and $\mathbf{b}$ convergence plot

Fig. 12 Irregular node distribution for elastic bar

as seen in Fig. 13. Here, nodal VCI methods give the best results. It should be noted that for uniform node distributions, all methods give essentially the same qualitative solution as $\mathrm{VC}$ constraints are in general met.

Now consider the same problem and integration methods with a transition in nodal spacing, as shown in Fig. 14. While errors in a static problem were found to be comparatively
Fig. 14 Node distribution with transition in spacing for elastic bar

small, for elastodynamics the transition in nodal spacing gives large errors for non-VC methods as shown in Fig. 15. Here it is seen that the VCI methods can provide much higher accuracy in both phase and amplitude compared to the variationally inconsistent methods.
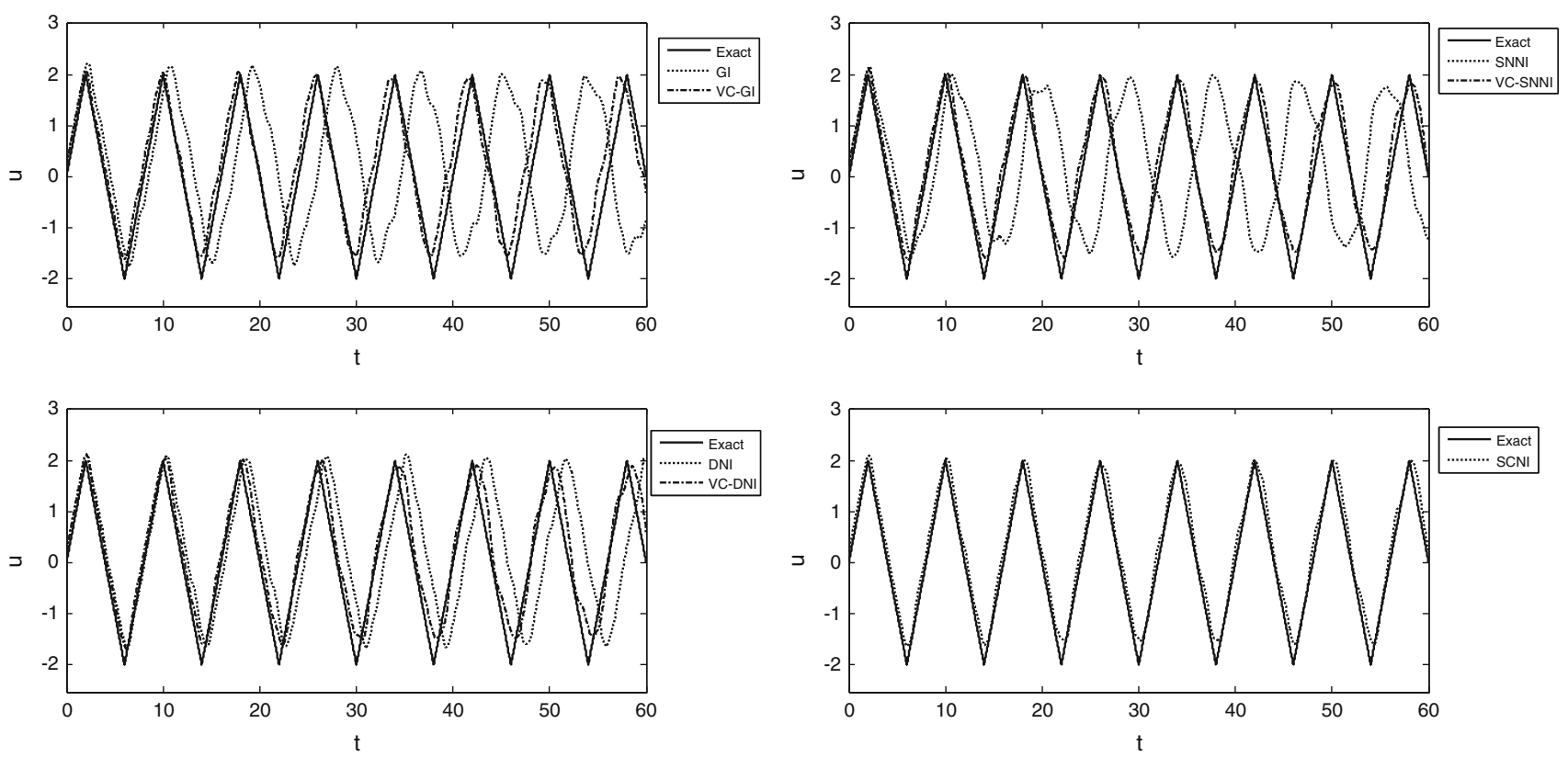

Fig. 13 Time histories for displacement at the free end of the bar with irregular node distribution 

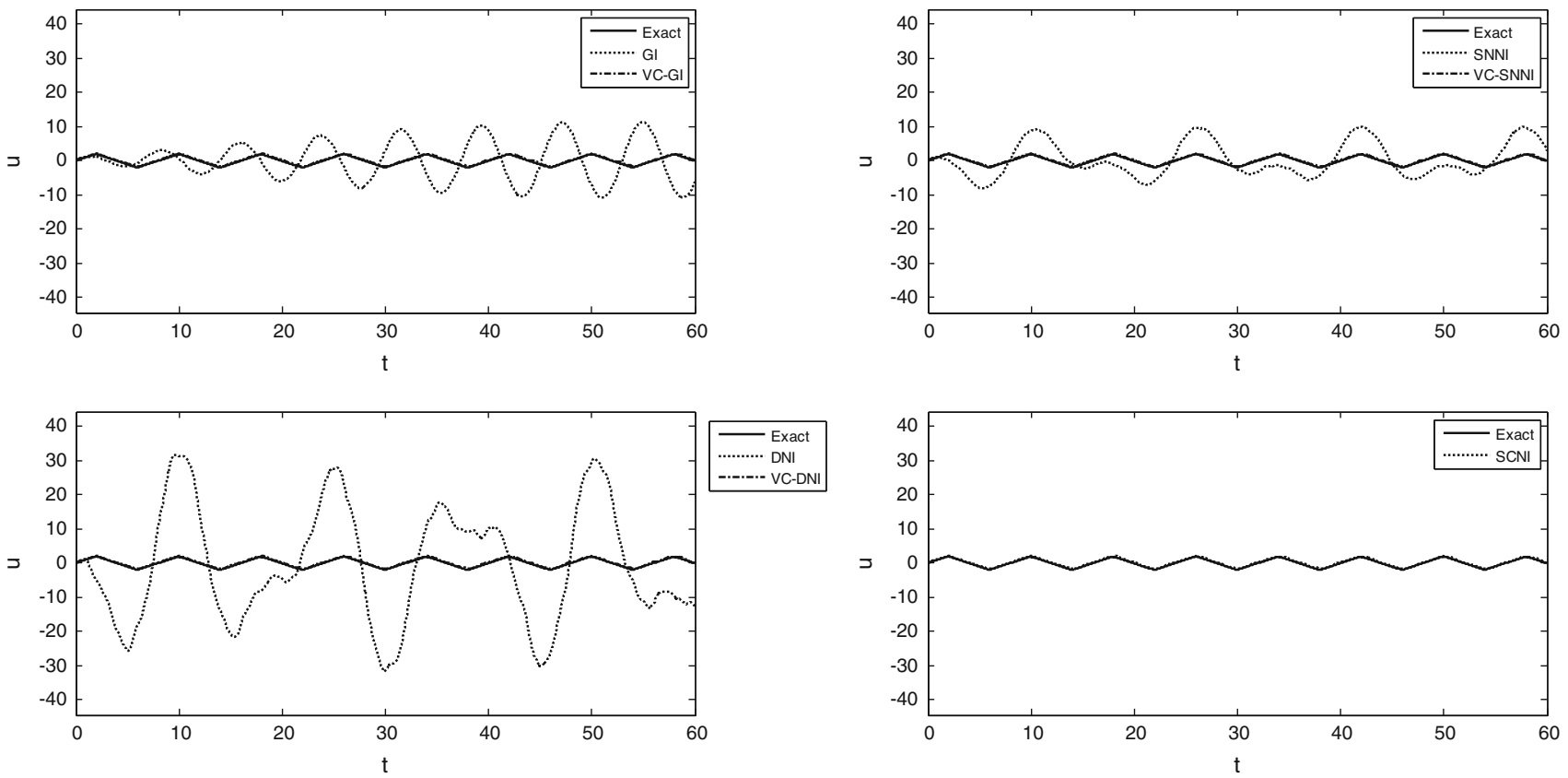

Fig. 15 Time histories for displacement and the free end of the bar with transition in nodal spacing (Fig. 14)

Table 3 Properties of aluminum bar

\begin{tabular}{ll}
\hline Young's modulus, $E$ & $78.2 \mathrm{GPa}$ \\
Poisson's ratio, $v$ & 0.3 \\
Density, $\rho$ & $2,700 \mathrm{~kg} / \mathrm{m}^{3}$ \\
Yield stress, $\sigma_{Y}$ & $0.29 \mathrm{GPa}$ \\
\hline
\end{tabular}

\subsection{Taylor bar impact}

Consider a cylindrical aluminum bar impacting a rigid frictionless wall with material properties shown in Table 3; a test first proposed by Taylor in [21] and performed in [24]. The initial height and radius of the bar are $2.346 \mathrm{~cm}$ and 0.391 $\mathrm{cm}$, respectively, and the initial velocity of the bar is 373.0 $\mathrm{m} / \mathrm{s}$.

For the constitutive model, $J_{2}$ plasticity with isotropic hardening is considered, where the yield function is given as

$$
f\left(\mathbf{s}, \bar{e}^{p}\right)=\|\mathbf{s}\|-\sqrt{\frac{2}{3}} K\left(\bar{e}^{p}\right)
$$

Here $\mathbf{s}$ is the deviatoric portion of the Cauchy stress, $\bar{e}^{p}$ is the equivalent plastic strain (EPS), and

$$
K\left(\bar{e}^{p}\right)=\sigma_{Y}\left(1+125 \bar{e}^{p}\right)^{0.1}
$$

Linear bases and quartic B-spline kernel functions are introduced in the RK approximation, and the nodal integration methods DNI, VC-DNI, SCNI, SNNI, and VC-SNNI are employed along with stabilized counterparts for the smoothed integration methods. Here, the matrix $\mathbf{C}$ in (3.8) was selected as the consistent tangent calculated at the nodal location.

The total deformations of the bar are similar for all the methods considered as shown in Table 4, but with stabilized methods giving less deformation for all cases. This is likely due to the slight increase in stiffness which can result from the contribution of the limiter term in (3.8). Experimental data is available only for the deformed height, so the finite element solution in [24] is also given as a reference. Overall, the results agree with the reference solutions provided, with stabilized methods agreeing the most. While the deformed heights and radii are fairly uniform, the instability due to nodal integration is apparent for the unstabilized, variationally inconsistent DNI and SNNI methods as shown in Fig. 16. Here the deformation is shown for the impact face with the EPS distribution, and "mesh" lines (used only for plotting) plotted to show the material deformation. It can also be seen that the VC corrected methods show superior stability over their uncorrected counterparts, with large improvements for both VC-DNI and VC-SNNI. The enhanced stability of the VCI methods agrees well with the eigenvalue analysis for non-uniform discretizations provided in Sect. 3.

The added stabilization for SNNI shows a large improvement in the pattern of deformation, whereas SCNI and VCSNNI show less of an improvement with stabilization. These results also agree with the results in Sect. 3, where only a marginal improvement is provided by stabilization when the solution by VCI methods is already stable. Comparing all the integration methods, it can be seen that MSCNI, MSNNI 


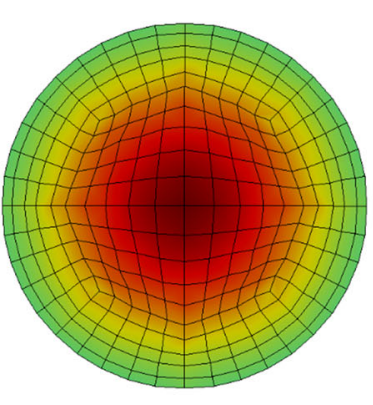

SCNI

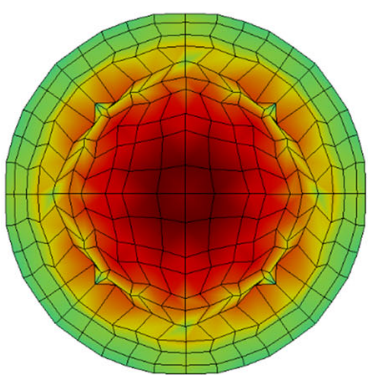

SNNI

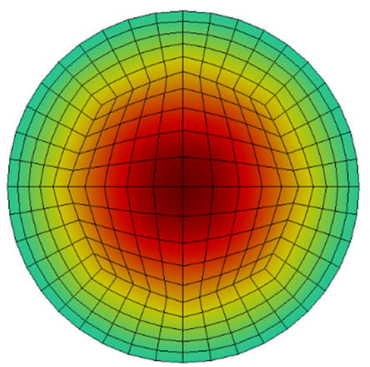

MSCNI

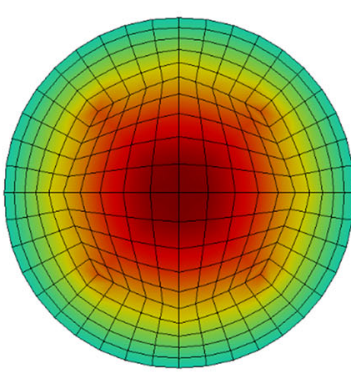

MSNNI
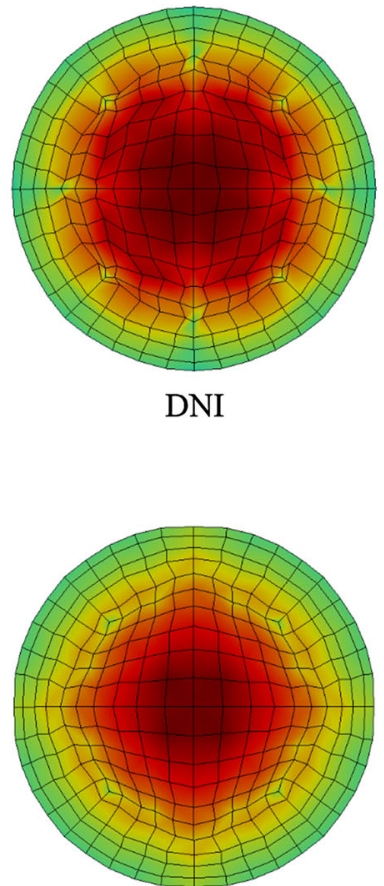

VC-SNNI

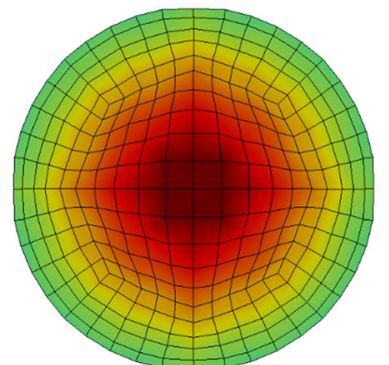

VC-DNI

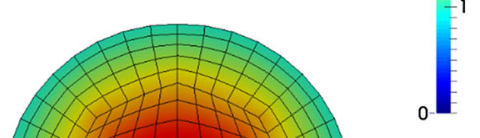

Fig. 16 Final deformation on the face of the Taylor bar for various nodal integration methods with EPS shown

Table 4 Dimensions of deformed Taylor bar

\begin{tabular}{lll}
\hline Method & Radius $(\mathrm{cm})$ & Height $(\mathrm{cm})$ \\
\hline SCNI & 0.782 & 1.631 \\
MSCNI & 0.754 & 1.635 \\
DNI & 0.781 & 1.633 \\
VC-DNI & 0.783 & 1.632 \\
SNNI & 0.786 & 1.637 \\
VC-SNNI & 0.784 & 1.637 \\
MSNNI & 0.754 & 1.638 \\
VC-MSNNI & 0.754 & 1.639 \\
HEMP [24] & 0.742 & 1.652 \\
Experimental[24] & - & 1.651 \\
\hline
\end{tabular}

and VC-MSNNI provide the best solutions, although VCDNI and VC-SNNI also perform well.

\section{Conclusions}

In this work it has been demonstrated that several commonly used domain integration methods can exhibit both instability and sub-optimal convergence due to inaccurate quadrature. Our particular interest is the improvement of the SNNI nodal integration method which provides greater simplicity for domain integration in fragment-impact problems, but suffers from low accuracy and instability.
To address both accuracy and stability, VCI methods with additional stabilization have been introduced. The VCI method recovers Galerkin exactness to an order consistent with the order of completeness in the approximation functions. The VCI methods are formulated under the assumed strain framework and can be conveniently enriched with strain energy stabilization for enhanced stability.

An eigenvalue analysis has been provided to show the enhanced stability of the proposed methods. Several numerical examples have been given to examine the performance of VCI methods with stabilization. For wave propagation problems, standard methods show large errors in phase and amplitude, while their variationally consistent counterparts do not. For large deformation impact problems, solutions for the VCI and VC corrected methods were also superior to their uncorrected counterparts, with stabilized variationally consistent methods (MSCNI and VC-MSNNI) showing the best performance.

\section{References}

1. Atluri SN, Shen S (1998) A new meshless local Petrov-Galerkin (MLPG) approach in computational mechanics. Comput Mech 22:117-127

2. Beissel S, Belytschko T (1996) Nodal integration of the elementfree Galerkin method. Comput Methods Appl Mech Eng 139:4974

3. Belytschko T, Lu YY, Gu L (1994) Element-free Galerkin methods. Int J Numer Methods Eng 37:229-256 
4. Chen JS, Hillman M, Rüter M (2013) An arbitrary order variationally consistent integration method for Galerkin meshfree methods. Int J Numer Methods Eng 95:387-418

5. Chen JS, Hu W, Puso M, Wu Y, Zhang X (2006) Strain smoothing for stabilization and regularization of Galerkin meshfree methods, vol. 57. In: Lecture notes computational science and engineering. pp. $57-76$

6. Chen JS, Pan C, Wu CT, Liu WK (1996) Reproducing kernel particle methods for large deformation analysis of non-linear structures. Comput Methods Appl Mech Eng 139:195-227

7. Chen JS, Wang D (2006) A constrained reproducing kernel particle formulation for shear deformable shell in Cartesian coordinate. Int J Numer Methods Eng 68:151-172

8. Chen JS, Wu Y, Guan PC, Teng H, Gaidos J, Hofstetter K, Alsaleh M (2009) A semi-Lagrangian reproducing kernel formulation for modeling earth moving operations. Mech Mater 41:670-683

9. Chen JS, Wu CT, Yoon S, You Y (2001) A stabilized conforming nodal integration for Galerkin mesh-free methods. Int J Numer Methods Eng 50:435-466

10. Chen JS, Yoon S, Wu CT (2002) Nonlinear version of stabilized conforming nodal integration for Galerkin meshfree methods. Int J Numer Methods Eng 53:2587-2615

11. De S, Bathe KJ (2000) The method of finite spheres. Comput Mech 24:329-345

12. Dolbow J, Belytschko $T$ (1999) Numerical integration of the Galerkin weak form in meshfree methods. Comput Mech 23:219230

13. Duan Q, Li X, Zhang H, Belytschko T (2012) Second-order accurate derivatives and integration schemes for meshfree methods. Int J Numer Methods Eng 92:399-424

14. Guan PC, Chi SW, Chen JS, Slawson TR, Roth MJ (2011) Semi-Lagrangian reproducing kernel particle method for fragmentimpact problems. Int J Impact Eng 38:1033-1047
15. Liu GR, Zhang GY, Wang YY, Zhong ZH, Li GY, Han X (2007) A nodal integration technique for meshfree radial point interpolation method (NI-RPIM). Int J Solids Struct 44:3840-3890

16. Liu WK, Jun S, Zhang YF (1995) Reproducing kernel particle methods. Int J Numer Methods Fluids 20:1081-1106

17. Liu Y, Belytschko T (2010) A new support integration scheme for the weakform in mesh-free methods. Int J Numer Methods Eng 82:699-715

18. Nayroles B, Touzot G, Villon P (1992) Generalizing the finite element method: diffuse approximation and diffuse elements. Comput Mech 10:307-318

19. Puso MA, Chen JS, Zywicz E, Elmer W (2008) Meshfree and finite element nodal integration methods. Int J Numer Methods Eng 74:416-446

20. Strang G, Fix G (2008) An analysis of the finite element method, 2nd edn. Wellesley-Cambridge Press, Massachusetts

21. Taylor G (1948) The use of flat-ended projectiles for determining dynamic yield stress, Part I. Proc R Soc Lond Ser A 194:289-299

22. Wang D, Chen JS (2004) Locking free stabilized conforming nodal integration for meshfree Mindlin-Reissner plate formulation. Comput Methods Appl Mech Eng 193:1065-1083

23. Wang D, Chen JS (2008) A Hermite reproducing kernel approximation for thin plate analysis with sub-domain stabilized conforming integration. Int J Numer Methods Eng 74:368-390

24. Wilkins ML, Guinan MW (1973) Impact of cylinders on rigid boundary. J Appl Phys 44:1200-1206

25. Yoo JW, Moran B, Chen JS (2004) Stabilized conforming nodal integration in the natural-element method. Int J Numer Methods Eng 60:861-890 